\title{
Opportunistic Source Coding for Data Gathering in Wireless Sensor Networks
}

\author{
Tao Cui \\ California Institute of Technology \\ MC 136-93 \\ Caltech \\ Pasadena, CA 91125 \\ cuitao52@hotmail.com
}

\begin{abstract}
We propose a jointly opportunistic source coding and opportunistic routing (OSCOR) protocol for correlated data gathering in wireless sensor networks. OSCOR improves data gathering efficiency by exploiting opportunistic data compression and multi-user diversity on wireless broadcast. OSCOR attacks challenges across network protocol layers by incorporating a slightly modified 802.11 MAC, a distributed source coding scheme based on Lempel-Ziv code and network coding, and a node compression ratio dependent metric combined with a modified Dijkstra's algorithm for path selection. We simulate OSCOR's performance and show it reduces the number of transmissions by nearly $25 \%$ compared with other schemes in small networks.
\end{abstract}

Copyright is held by author/owner(s)

Tapia'07, October 14-17, 2007, Lake Buena Vista, Florida, USA ACM 978-1-59593-866-4/07/0010 